\title{
Setting up an adult congenital heart programme
}

Gary D. Webb and Gruschen R. Veldtman

Cincinnati Adolescent and Adult Congenital Heart Program,

Cincinnati Children's Hospital Heart Institute, Cincinnati, Ohio,

United States of America

Address for correspondence:

Dr Gary D.Webb

Cincinnati Children's Hospital Heart Institute,

3333 Burnet Avenue

Cincinnati

Ohio

45229

United States of America

Email: gary.webb@cchmc.org

As the number of adult patients with congenital heart defects continues to increase, the challenge of establishing appropriate care for them is being faced throughout the developed world. We both worked at the oldest adult congenital heart disease (ACHD) or grown up heart disease $(\mathrm{GUCH})$ programme in the world, the Toronto Congenital Cardiac Center for Adults, established in 1959. This was a very mature and large programme, one that took many years to build. One of us (GW) has since had the opportunity to start building two programmes in large American institutions from a small base. This will be similar to the challenge being faced in most locations. One of us (GRV) had the opportunity to grow and develop a regional ACHD/GUCH programme in Southampton, UK. We are now both working in Cincinnati. We will do our best to share the lessons that we and others have learned in the hopes that it will help our colleagues understand what they can or must do. (1-5) In so doing, we will address our remarks to an imaginary professional colleague interested in establishing an ACHD service.

\section{PRACTICAL CONSIDERATIONS}

The first challenge has to do with understanding where the patients are going to come from.

Adult CHD patients may come from paediatric cardiologists in the community, as well as from adult cardiologists and other sources. It's important to know before you set up your new ACHD programme how much support you will have from these sources, and

\section{ABSTRACT}

With the growing number of adult patients with congenital heart defects in the developing world, many institutions and practice groups are beginning or growing their adult congenital heart disease (ACHD) programmes. In this paper, we review:

- Practical considerations in establishing a clinic (Where will your patients come from? How many new referrals annually can you anticipate?);

- Transition and transfer challenges (can you avoid the loss to care of children with moderate and complex congenital heart defects? Is there an agreed age of transfer policy? Is there an available and effective transition process?);

- Institutional considerations (do you really have the support of your institution? Where should your program be located? Where will you admit your inpatients?);

- Patient care imperatives (how will you commit to excellent patient care? How will you meet the needs of your patients? Clearly designate which patients you will need to refer out and to which services they will be referred);

- The composition of the ACHD team (begin with two ACHD cardiologists and skilled echocardiography, consider a mix of pediatric trained and adult trained cardiologists, grow from there in a modular fashion if you can to include more multidisciplinary team members, and duplicate essential service positions); and

- Organisation and accreditation of ACHD care (ACHD services need to be managed differently than pediatric services across the full diagnostic and treatment clinical spectrum). SAHeart 20 13; 0:586-59|

how many patients you can expect to be referred. To some extent, you may be able to gain referrals from primary care physicians or self-referrals from patients and family members, but this strategy calls for a much broader marketing effort, and will consume much more time.

In some countries, establishing referral protocols and infrastructures from primary and secondary care level hospitals that have limited or no expertise in ACHD, will be essential to the development and growth of the programme.

The second possible source of patients is new graduates from paediatric cardiology practices. If you have the support of a group 
of paediatric cardiologists who will agree to transfer their newly adult patients to you, you have a great advantage. If you don't have the support of the paediatric cardiology community in your area, you have a real problem.

\section{How many patients are needed to build a strong ACHD programme?}

In our opinion, if your aim is to establish a comprehensive multidisciplinary ACHD programme, you need approximately 250 new referrals a year, or a base of at least I 500 patients in order to develop and maintain team skills, to train fellows, and drive adequate volumes in both diagnostic and interventional arenas.

\section{TRANSITION AND TRANSFER CHALLENGES}

A related issue has to do with an agreed age at transfer policy. In Canada, transfer from paediatric to adult practice occurs at age 18 . In the United Kingdom, transfer tends to be even younger, particularly in adult institutions that do not also have paediatric cardiology on-site. In the United States, such clear age criteria are rare, but an agreed age of transfer policy is increasingly needed in those locations where high-quality ACHD programmes are available in the USA.

Another important issue has to do with avoiding loss to care of children with moderate and complex congenital heart conditions. There is ample evidence in the literature that patients who need lifelong care and surveillance nonetheless drop out of follow-up at all ages. ${ }^{(6)}$ The main initiative to keep these patients in care is the responsibility of the paediatric practitioners and institutions. In our opinion, patients with moderate and complex congenital heart conditions need to be identified clearly as needing lifelong surveillance, and efforts should be made in the paediatric practices to follow up on missed appointments and make sure that patients are receiving appropriate care and surveillance. Once they reach adult age, the same effort needs to be made from the ACHD clinic or practitioner, especially since we know the human brain does not fully mature until approximately age 25 .

An important initiative to help ensure that young people are not lost to care inappropriately is an effective transition process. The main point of this process is to encourage young people to take appropriate responsibility for their own health. Healthy attitudes and behaviors are encouraged. Young people are encouraged to begin to progressively behave more like adults. Developmental tasks include gradually becoming independent from parents, developing a healthy self-image, creating educational and vocational goals, becoming financially independent, living independently, becoming involved in a marriage or partnership, participation in the community, and all this in the presence of good mental health. The transition process should occur ideally in association with each regular adolescent clinical visits beginning at age 12 . Issues include career planning, lifestyle choices, insurance planning and reproductive counseling. We know that young people who make good lifestyle choices are more likely to take responsibility for their own health and lives, so healthy lifestyles should be strongly encouraged.

Some patients can be considered as "low risk graduates" of paediatric care. Some have really been cured of their original condition. Some are unlikely ever to have problems. Many of these will have a normal life expectancy. Almost all will have no limitations on exercise and lifestyle. Indeed, some will not even need specialised care. Nonetheless, even low risk patients (such as those with repaired pulmonary stenosis, closed secundum ASDs, and small VSDs) need to learn of their favorable expectations and their responsibilities from their caregivers. The same facts need to be conveyed to their parents. It is important that a clear message has been received. Transfer of these low risk graduates is usually easy since good primary care physicians and cardiologists are usually available and appropriate to care for them, and since team care is seldom required.

By contrast, "medium risk to high risk graduates" will need expert lifelong surveillance. Few are cured. Some may never have problems. Some will have a normal life expectancy. Nonetheless, group life expectancy is clearly reduced in a lesion-dependent fashion. Many of these patients will have limitations of their exercise capacity and lifestyle. Further problems can be anticipated in most. All of these patients will need specialised surveillance and sometimes care. Examples of moderately complex patients are atriventricular septal defects, aortic coarctation, Ebstein anomaly, arterial switch patients, repaired tetralogy of Fallot, and moderate to severe pulmonary valve lesions. Examples of very complex patients include patients with cyanotic congenital heart disease, severe pulmonary hypertensive patients, Fontan patients, Mustard/Senning patients, patients with conduits, and those with truncus arteriosus. Such patients also need clear information on their responsibilities to look after themselves and to remain in expert surveillance. Their caregivers need to instruct them when they reach transfer age on who to see, when to see them, and why they need expert surveillance. They also need to know how to identify expert care, and where to find it.(7) 
Part of the transition process includes educating the patient and the family. What condition does the patient have? What treatment has he/she received? What are the future expectations for the patient? Are there any threats that can be anticipated or any precautions that should be taken? What symptoms should be watched for? How frequently should the patient be seen, and by whom and where?

\section{INSTITUTIONAL CONSIDERATIONS}

You will also need the support of your institution and/or your cardiology division/practice group. Is your institution or group prepared to give you a long-term commitment to build and maintain the programme, and to providing excellent care of $\mathrm{ACHD}$ patients? If so, there needs to be in agreement on goals, objectives, timelines, financial considerations, and other issues.

Where should your programme be located? An adult hospital? A children's hospital? There is no clear correct answer to this question. It is safe to say that the ideal situation would be one in which both adult and paediatric services are provided in the same institution. There is evidence that successful transfer to adult care is more effective and successful when the patient can be transferred within the same institution. ${ }^{(8,9)}$ The availability of both adult and paediatric providers also improves your potential flexibility in identifying the right resources for your particular patient, regardless of whether the resources come from the paediatric or the adult world.

In our experience, having an ACHD clinic in an adult hospital means you may lose the opportunity to keep complex CHD children and adolescents in care unless you have remarkable support from your paediatric cardiologists. Furthermore, having an ACHD clinic in an adult hospital means you may not be able to negotiate the smooth transfer of complex CHD kids and adolescents to adult care. By contrast, having an ACHD clinic in a children's hospital may help you avoid the loss to care of complex CHD kids and adolescents and help affect a smooth transfer to adult care. (Editors comment: The argument for having the ACHD clinic in an adult hospital includes the fact that caring for adults in an adult environment provides the adult culture that young adults need to complete their transition to independent adulthood. Pregnancy is also more comfortably dealt with in an adult setting, and as patients age and develop acquired co-morbidities, the adult setting provides the most appropriate care.)
Whether your programme is located in an adult hospital or a children's hospital, you should be aware of the important cultural differences between these two systems. Considerable time and effort is likely to be required to overcome cultural disparities. Team members with an adult background will align most easily with an adult environment, and team members with a paediatric background will align best with a paediatric environment. The reverse also applies, and the process of alignment may well be painful.

Deciding where to deliver outpatient ACHD care is a relatively straightforward one from a practical standpoint. On the other hand, a paediatric outpatient location may well highlight the need to have separate and distinct protocols for adult patients as regards echocardiography, exercise testing, and other diagnostic modalities as well as patient care challenges. The ambulatory challenge pales in comparison to the decision to admit adult patients to a children's hospital! Substantial staff push back can be anticipated, demonstrating the clear need for staff members with adult training to form most of the care team for adult inpatients.

In countries where healthcare is nationalised, the importance of engaging the governmental health department's interest and support cannot be underestimated in helping to establish and maintain a well-organised service.

\section{PATIENT CARE IMPERATIVES}

You should commit yourself and your programme to excellent patient care. This will reduce the number of times you do things you shouldn't because of pride or self-interest. Ultimately, adhering to this principle will help you build and maintain your credibility.

Your programme's main mission should be to be able to address the needs of any patient that comes through your door. You may or may not be able to do everything in your own institution or community. If not, you need to make sure that services of high quality are available to your patients, and you should refer them to the best available resources. You will need to be clear on what services you will be able to provide locally, and on what services you will need to refer out. The distribution of these service arrangements may change with time, and almost all programmes will have to refer some patients externally in the interests of best patient care. It goes without saying that once you establish a programme, care needs to be available at all times. 


\section{THE ACHD TEAM}

The members of a multidisciplinary ACHD team are many (Figure I). Moreover, you don't just want to have one person in each of your key categories. You should take pains to at least duplicate key skills.

You need at least two ACHD cardiologists. These can come from either an adult cardiology or a paediatric cardiology background. If from paediatric cardiology, that person needs to practice differently when dealing with adults than when dealing with children and their parents. Ideally, both types of cardiologists will have received specific training in adult congenital heart disease. If not, there is at least the need for a clear career commitment to become an excellent ACHD cardiologist, and to grow and maintain one's skills.

In general, we believe it's important to have a mix of adult-trained and paediatric-trained cardiologists in the ACHD clinic. Regardless of how good and well trained the cardiologists are, it's highly likely that collaboration with a colleague with the opposite training will end up being a net positive for the clinic and for the patients.

As you are beginning the programme, you'll need to identify potential consulting team members. You'll need to understand both their abilities and limitations. You will need to develop and improve the capabilities of your consulting team in a realistic and constructive way.

You definitely need an excellent echocardiography service. This will include both physicians and sonographers. The quality of the work is critically important. It should be noted that adult echoes and paediatric echoes are very different, and are reported differently.

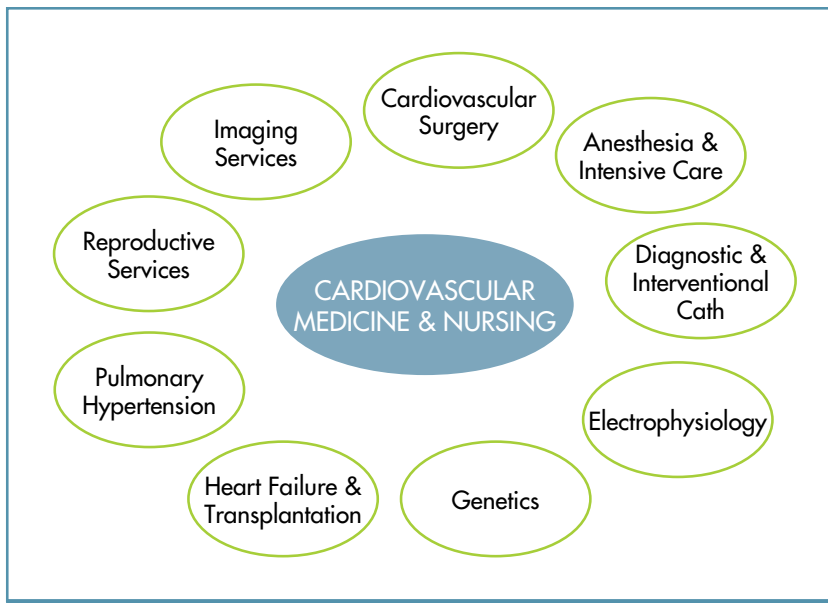

FIGURE I: Major members of a multidisciplinary ACHD team.
Adult approaches are needed for adult patients. Ideally, a full range of echo services will be provided.

The combination of two ACHD cardiologists and an excellent echocardiography service will form the essential elements of a small ACHD practice. Other services can either be accessed elsewhere, or included within the programme.

Excellent nursing support adds value to the team. Whether nursing team members should come from an adult or paediatric background can be debated. Their contributions to patient care and programme activities depend upon their particular qualifications and the local regulatory environment.

Access to a strong congenital heart surgical programme is essential. This includes the entire surgical team including surgeons, anaesthetists, perfusionists, intensivists, nurses, and many others. The most common surgical procedure done in ACHD patients is pulmonary valve replacement. In our opinion, these are best handled by congenital heart surgeons. There are other patients that can be handled by adult cardiovascular surgeons skilled at aortic, mitral and aortic valve surgery, coronary artery bypass, and so on. If the ACHD programme is going to include patients with Marfan syndrome, there needs to be 24/7 availability of emergency surgical services to deal with anticipated aortic dissections. If the ACHD programme is going to include patients with severe pulmonary hypertension, special resources also need to be available to manage these contingencies. As the If the programme is going to include cardiac patients who are pregnant, appropriate resources must be available.

Access to excellent diagnostic and interventional catheterisation services also need to be available. The cardiologists doing these procedures most often will come from a paediatric cardiology background, although adult cardiologists with training in structural heart disease may well be capable of managing many catheterisation cases. In either instance, the interests of the patient should be primary. The procedure should be done by someone with appropriate expertise and experience and whose equipment and resources offer the patient the best possible chance of an excellent outcome. Paediatric cardiac catheterisation labs are biplane, and biplane facilities are seldom available in the adult world. Ideally, biplane equipment would be used for both diagnostic and interventional purposes on ACHD patients.

Access to excellent electrophysiology services are also essential. $\mathrm{ACHD}$ patients commonly have EP issues. Again, it's not easy to say 
whether the best person for a given patient would come from a paediatric EP or an adult EP background. The choice of the consultant again will need to be individualised.

Access to heart failure and transplant services will also need to be available. As patient numbers grow and as patients age, this becomes an increasing issue in an ACHD programme. Inevitably, access to pulmonary hypertension resources will also be needed. Many $\mathrm{ACHD}$ programmes now include team members with pulmonary hypertension expertise.

Reproductive services need to be available locally. This begins with the ability to counsel women of childbearing age regarding contraceptive options and their pregnancy risks. As a rule, one or more interested gynecologists may well make a valuable contribution to the family planning aspects of ACHD patient care. Alternatively, a good primary care physician might provide many of the same services. The availability of maternal fetal medicine services is also important. The ACHD programme should provide the cardiac component of surveillance and treatment planning in collaboration with one or more obstetrical teams.

Imaging services are another essential resource. Excellence in MRI is extremely important, and CT angiography will also need to be available. Specific training and experience in congenital heart disease and/or ACHD is essential if major errors are to be avoided and maximum information obtained.

There are any number of additional people who might be important from time to time. Fontan patients often have liver problems, and a knowledgeable hepatologist may be very important. Many ACHD patients have chronic renal issues, so nephrology support and involvement could be very important. Genetics in the form of a geneticist and a genetics counselor may also be very important in the assessment and management of some patients. Hematology support may be required at times. Mental health resources are an important part of a comprehensive service. In our own practice in Cincinnati, we have found it very valuable to include internists/ paediatricians who have trained in both paediatrics and internal medicine. These colleagues can provide adult inpatient support, primary care within our cardiac clinic to patients, improving communications around patient care while also taking a lead role in our transition programme.

In starting a new ACHD programme, you need to have the essential elements in place before you open the door. Once you do open the door and patients come in in sufficient numbers, your team will gain additional clinical experience and will become even more talented than they were at the outset. As one spreads this experience across the various members of the local and outreach teams, the depth of experience and knowledge deepens and broadens and care is provided in a more consistent and effective way. In turn, this experience leads to the generation of new knowledge. The team would be well advised to focus on quality outcome metrics in order to ensure themselves that they are maintaining standards and following published guidelines in the care of their patients. As the team gains experience, it will teach others who will then hopefully make a career commitment to continue the growth of the excellent resources available to the care of our patients.

\section{ORGANISATION AND ACCREDITATION OF ACHD CARE}

The Adult Congenital Heart Association in the USA is in the process of creating accreditation metrics for American ACHD clinics. This is an important initiative that will help those of us working in the United States to focus on the various quality measures that we need to meet.

In the United Kingdom, the National Health Service has defined three different levels of care for ACHD patients and has defined the roles and responsibilities for each of those three levels. To our knowledge, they have not yet been published. In Canada, ACHD services are provided at Canadian medical schools, and so care of patients is reasonably well organised.

We believe that when paediatric cardiologists are going to have a substantial profile in ACHD care, they need to make a career commitment to the ACHD patient, and have a different agenda for their young adult and adult patients than they have for their paediatric and adolescent patients.

ACHD services need to be designed separately from paediatric cardiology services and from adult cardiology services. This applies to a wide range of activities including echocardiography, exercise testing, cardiac catheterisation, and inpatient care. This may prove to be a difficult process, but we believe that extending paediatric services into the adult age range is unlikely to be successful, as is extending services for acquired heart disease into the congenital heart population. We believe that, in most cases, parallel systems in all these dimensions should be created. 


\section{CONCLUSION}

So in summary, to build a strong ACHD programme, you will need: lots of suitable patients; a supportive institution; supportive colleagues; a talented team; a commitment to excellent patient care; educated patients and families; an effective transition process; and a clear transfer/graduation policy. You will focus mainly on medium-risk to high-risk ACHD patients, and will work to keep them in care and to meet whatever health needs they may develop. You will need to decide what type of institution to locate your programme in. Most of your care will be ambulatory, and you will need to establish suitable and different diagnostic and management protocols for the ACHD patients regardless of whether you are in a paediatric institution or an adult facility. If you are based in a children's hospital, the admission of adult patients will be trying until you develop an appropriately adult-trained care team to take the load off the paediatric practitioners. You will need to keep the interests of the patient uppermost in your planning, and will need to be clear what services you will be able to provide locally, and which services will need to be accessed elsewhere. You will be challenged to develop your multidisciplinary care team, and this will take both patience and persistence. We believe that collaboration of at least one paediatric-trained cardiologist with at least one adult-trained cardiologist is a good foundation for a programme. An excellent echocardiography service is also imperative, and will need to be different from both paediatric echo services and adult acquired heart disease echo services. In time, we expect that our practices will become more and more evidence-based, and that appropriate accreditation criteria will be developed for us to meet. It won't be easy, but there are a growing number of patients who are depending on us to get the job done, and to get it done well.

\section{REFERENCES}

I. Child JS, et al. (200I). Task force 3: Workforce description and educational requirements for the care of adults with congenital heart disease. J Am Coll Cardiol 37(5): 1 | 83-1 | 87

2. Foster $E$, et al. (200I). Task force 2: Special health care needs of adults with congenital heart disease. J Am Coll Cardiol 37(5): I 176-। 183.

3. Landzberg MJ, et al. (200I). Task force 4: Organisation of delivery systems for adults with congenital heart disease. J Am Coll Cardiol 37(5): I I87-1 193.

4. Skorton DJ, et al. (200I). Task force 5: Adults with congenital heart disease: access to care. J Am Coll Cardiol 37(5): I 193-1 198.

5. Warnes C A, et al. (200I). Task force I: The changing profile of congenital heart disease in adult life. J Am Coll Cardiol 37(5): I 170- I 175.

6. Mackie AS, et al. (2009). Children and adults with congenital heart disease lost to follow-up: who and when? Circulation 120(4):302-309.

7. Reid G], et al. (2004). Prevalence and correlates of successful transfer from paediatric to adult health care among a cohort of young adults with complex congenital heart defects. Paediatrics II3(3 Pt I):e197-205.

8. Goossens E, et al. (201 I). Transfer of adolescents with congenital heart disease from paediatric cardiology to adult health care: an analysis of transfer destinations. J Am Coll Cardiol 57(23):2368-2374.

9. Norris MD, et al. (2013). Prevalence and patterns of retention in cardiac care in young adults with congenital heart disease. J Pediatr I63(3):902-904 e90।.

\section{Conflict of interest: none declared.}

